\title{
GEODYNAMO: NUMERICAL SIMULATIONS
}

\author{
Gary A. Glatzmaier
}

\section{Introduction}

The geodynamo is the name given to the mechanism in the Earth's core that maintains the Earth's magnetic field (see The Geodynamo). The current consensus is that flow of the liquid iron alloy within the outer core, driven by buoyancy forces and influenced by the Earth's rotation, generates large electric currents that induce magnetic field, compensating for the natural decay of the field. The details of how this produces a slowly changing magnetic field that is mainly dipolar in structure at the Earth's surface, with occasional dipole reversals, has been the subject of considerable research by many people for many years.

The fundamental theory, put forward in the 1950's, is that differential rotation within the fluid core shears poloidal (north-south and radial) magnetic field lines into toroidal (east-west) magnetic field; and three-dimensional (3D) helical fluid flow twists toroidal field lines into poloidal field. The more sheared and twisted the field structure the faster it decays away; that is, magnetic diffusion (reconnection) continually smooths out the field. The field is self-sustaining if, on average, the generation of field is balanced by its decay. Discovering and understanding the details of how rotating convection in Earth's fluid outer core maintains the observed intensity, structure and time dependencies requires 3D computer models of the geodynamo.

Magnetohydrodynamic (MHD) dynamo simulations are numerical solutions of a coupled set of nonlinear differential equations that describe the 3D evolution of the thermodynamic variables, the fluid velocity and the magnetic field. Because so little can be detected about the geodynamo, other than the poloidal magnetic field at the surface (today's field in detail and the paleomagnetic field in much less detail) and what can be inferred from seismic measurements and variations in the length of the day and possibly in the gravitational field, models of the geodynamo are used as much to predict what has not been observed as they are used to explain what has. When such a model generates a magnetic field that, at the model's surface, looks qualitatively similar to the Earth's surface field in terms of structure, intensity and time-dependence, then it is plausible that the 3D flows and fields inside the model core are qualitatively similar to those in the Earth's core. Analyzing this detailed simulated data provides a physical description and explanation of the model's dynamo mechanism and, by assumption, of the geodynamo.

The first 3D global convective dynamo simulations were developed in the 1980's to study the solar dynamo. Gilman and Miller [1981] pioneered this style of research by constructing the first 3D MHD dynamo model. However, they simplified the problem by specifying a constant background density, i.e., they used the Boussinesq approximation of the equations of motion. Glatzmaier [1984] developed a 3D MHD dynamo model using the anelastic approximation, which accounts for the stratification of density within the sun. Zhang and Busse [1988] used a 3D model to study the onset of dynamo action within the Boussinesq approximation. However, the first MHD models of the Earth's dynamo that successfully produced a time dependent and dominantly dipolar field at the model's 
surface were not published until 1995 [Kageyama et al., 1995; Jones et al., 1995; Glatzmaier and Roberts, 1995]. Since then several groups around the world have developed dynamo models and several others are currently being designed. Some features of the various simulated fields are robust, like the dominance of the dipolar part of the field outside the core. Other features, like the 3D structure and time dependence of the temperature, flow and field inside the core, depend on the chosen boundary conditions, parameter space and numerical resolution. Many review articles have been written that describe and compare these models [e.g., Hollerbach, 1996; Glatzmaier and Roberts, 1997; Fearn, 1998; Busse, 2000; Roberts and Glatzmaier, 2000; Dormy et al., 2000; Christensen et al., 2001; Busse, 2002; Kono and Roberts, 2002; Glatzmaier, 2002].

\section{Model description}

Models are based on equations that describe fluid dynamics and magnetic field generation (see Boussinesq and anelastic approximations). The equation of mass conservation is used with the very good assumption that the fluid flow velocity in the Earth's outer core is small relative to the local sound speed. The anelastic version of mass conservation accounts for a depth-dependent background density; the density at the bottom of the Earth's fluid core is about $20 \%$ greater than that at the top. The Boussinesq approximation simplifies the equations further by neglecting this density stratification, i.e., by assuming a constant background density. An equation of state relates perturbations in temperature and pressure to density perturbations, which are used to compute the buoyancy forces, which drive convection. Newton's second law of motion (conservation of momentum) determines how the local fluid velocity changes with time due to buoyancy, pressure gradient, viscous, rotational (Coriolis) and magnetic (Lorentz) forces. The magnetohydrodynamic equations (i.e., Maxwell's equations and Ohm's law with the extremely good assumption that the fluid velocity is small relative to the speed of light) describe how the local magnetic field changes with time due to induction by the flow and diffusion due to finite conductivity. The second law of thermodynamics dictates how thermal diffusion and Joule and viscous heating determine the local time rate of change of entropy (or temperature). Additional equations are sometimes included that account for perturbations in composition and gravitational potential and their effects on buoyancy.

This set of coupled nonlinear differential equations, with a set of prescribed boundary conditions, is solved each numerical time step to obtain the evolution in 3D of the fluid flow, magnetic field and thermodynamic perturbations. Most geodynamo models have employed spherical harmonic expansions in the horizontal directions and either Chebyshev polynomial expansions or finite differences in radius. The equations are integrated in time typically by treating the linear terms implicitly and the nonlinear terms explicitly. The review articles mentioned above describe the variations on the equations, boundary conditions and numerical methods employed in the various models of the geodynamo.

\section{Current results}

Since the mid 1990's 3D computer simulations have advanced our understanding of the geodynamo. The simulations show that a dominantly dipolar magnetic field, not unlike the Earth's, can be maintained by convection driven by an Earth-like heat flux. A typical 
snapshot of the simulated magnetic field from a geodynamo model is illustrated in Figure 1 with a set of field lines. In the fluid outer core, where the field is generated, field lines are twisted and sheared by the flow. The field that extends beyond the core is significantly weaker and dominantly dipolar at the model's surface, not unlike the geomagnetic field. For most geodynamo simulations, the non-dipolar part of the surface field, at certain locations and times, propagates westward at about $0.2^{\circ}$ per year as has been observed in the geomagnetic field over the past couple hundred years.

Several dynamo models have electrically conducting inner cores that on average drift eastward relative to the mantle [e.g., Glatzmaier and Roberts, 1995; Sakuraba and Kono, 1999; Christensen et al. 2001], opposite to the propagation direction of the surface magnetic field. Inside the fluid core the simulated flow has a "thermal wind" component that, near the inner core, is predominantly eastward relative to the mantle. Magnetic field in these models that permeates both this flow and the inner core tries to drag the inner core in the direction of the flow. This magnetic torque is resisted by a gravitational torque between the mantle and the topography on the inner core surface. The amplitude of the super-rotation rate predicted by geodynamo models depends on the model's prescribed parameters and assumptions and on the very poorly constrained viscosity assumed for the inner core's deformable surface layer, which by definition is near the melting temperature. The original prediction was an average of about $2^{\circ}$ longitude per year faster than the surface. Since then the super-rotation rate of the Earth's inner core (today) has been inferred from several seismic analyses, but is still controversial. There is a spread in the inferred values, from the initial estimates of $1^{\circ}$ to $3^{\circ}$ eastward per year (relative to the Earth's surface) to some that are zero to within an uncertainty of $0.2^{\circ}$ per year. More recent geodynamo models that include an inhibiting gravitational torque also predict smaller super-rotation rates.

On a much longer time scale, the dipolar part of the Earth's field occasionally reverses (see Reversals, theory). The reversals seen in the paleomagnetic record are non-periodic. The times between reversals are measured in hundreds of thousands of years; whereas the time to complete a reversal is typically a few thousand years, less than a magnetic dipole decay time. Several dynamo simulations have produced spontaneous non-periodic magnetic dipole reversals [Glatzmaier and Roberts, 1995; Sarson and Jones, 1999; Kageyama et al., 1999; Glatzmaier et al., 1999; Kutzner and Christensen, 2002]. Regular (periodic) reversals, like the dynamo-wave reversals seen in early solar dynamo simulations, have also occurred in recent dynamo simulations.

One of the simulated reversals is portrayed in Figure 2 with four snapshots spanning about 9,000 years. The radial component of the field is shown at both the core-mantle boundary $(\mathrm{CMB})$ and the surface of the model Earth. The reversal, as viewed in these surfaces, begins with reversed magnetic flux patches in both the northern and southern hemispheres. The longitudinally-averaged poloidal and toroidal parts of the field inside the core are also illustrated at these times. Although when viewed at the model's surface the reversal appears complete by the third snapshot, another three thousand years is required for the original field polarity to decay out of the inner core and the new polarity to diffuse in.

Small changes in the local flow structure continually occur in this highly nonlinear chaotic system. These can generate local magnetic anomalies that are reversed relative to 
the direction of the global dipolar field structure. If the thermal and compositional perturbations continue to drive the fluid flow in a way that amplifies this reversed field polarity while destroying the original polarity, the entire global field structure would eventually reverse. However, more often the local reversed polarity is not able to survive and the original polarity fully recovers because it takes a couple of thousand years for the original polarity to decay out of the solid inner core. This is a plausible explanation for "events", which occur when the paleomagnetic field (as measured at the Earth's surface) reverses and then reverses back, all within about ten thousand years.

On an even longer time scale, the frequency of reversals seen in the paleomagnetic record varies. The frequency of non-periodic reversals in geodynamo simulations has been found to depend on the pattern of outward heat flux imposed over the CMB (presumably controlled in the Earth by mantle convection) and on the magnitude of the convective driving relative to the effect of rotation.

Many studies have been conducted via dynamo simulations to, for example, assess the effects of the size and conductivity of the solid inner core, of a stably stratified layer at the top of the core, of heterogeneous thermal boundary conditions, of different velocity boundary conditions and of computing with different parameters. These models differ in several respects. For example, the Boussinesq instead of the anelastic approximation may be used, compositional buoyancy and perturbations in the gravitational field may be neglected, different boundary conditions and spatial resolutions may be chosen, the inner core may be treated as an insulator instead of a conductor or may not be free to rotate. As a result, the simulated flow and field structures inside the core differ among the various simulations. For example, the strength of the shear flow on the "tangent cylinder" (the imaginary cylinder tangent to the inner core equator; Figure 1), which depends on the relative dominance of the Coriolis forces, is not the same for all simulations. Likewise, the vigor of the convection and the resulting magnetic field generation tends to be greater outside this tangent cylinder for some models and inside for others. But all the solutions have a westward zonal flow in the upper part of the fluid core and a dominantly dipolar magnetic field outside the core.

When assuming Earth values for the radius and rotation rate of the core, all models of the geodynamo have been forced (due to computational limitations) to use a viscous diffusivity that is at least three to four orders of magnitude larger than estimates of what a turbulent (or eddy) viscosity should be (about $2 \mathrm{~m}^{2} / \mathrm{s}$ ) for the spatial resolutions that have been employed. In addition to this enhanced viscosity, one must decide how to prescribe the thermal, compositional and magnetic diffusivities. One of two extremes has typically been chosen. These diffusivities could be set equal to the Earth's actual magnetic diffusivity $\left(2 \mathrm{~m}^{2} / \mathrm{s}\right)$, making these much smaller than the specified viscous diffusivity; this was the choice for most of the Glatzmaier-Roberts simulations. Alternatively, they could be set equal to the enhanced viscous diffusivity, making all (turbulent) diffusivities too large, but at least equal; this was the choice of most of the other models. Neither choice is satisfactory.

\section{Future challenges}

Because of the large turbulent diffusion coefficients, all geodynamo simulations have 
produced large-scale laminar convection. That is, convective cells and plumes of the simulated flow typically span the entire depth of the fluid outer core, unlike the small-scale turbulence that likely exists in the Earth's core.

The fundamental question about geodynamo models is how well do they simulate the actual dynamo mechanism of the Earth's core. Some geodynamo modelers have argued, or at least suggested, that the large (global) scales of the temperature, flow and field seen in these simulations should be fairly realistic because the prescribed viscous and thermal diffusivities may be asymptotically small enough. For example, in most simulations, viscous forces (away from the boundaries) tend to be $10^{4}$ times smaller than Coriolis and Lorentz forces. Other modelers are less confident that current simulations are realistic even at the large scales because the model diffusivities are so large. Only when computing resources improve to the point where we can further reduce the turbulent diffusivities by several orders of magnitude and produce strongly turbulent simulations will we be able to answer this fundamental question.

In the mean time, we may be able to get some insight from very highly resolved two-dimensional simulations of magnetoconvection. These simulations can use diffusivities a thousand times smaller than those of the current 3D simulations. They demonstrate that strongly turbulent $2 \mathrm{D}$ rotating magnetoconvection has significantly different spatial structure and time dependence than the corresponding 2D laminar simulations obtained with much larger diffusivities.

These findings suggest that current 3D laminar dynamo simulations may be missing critical dynamical phenomena. Therefore it is important to strive for much greater spatial resolution in 3D models in order to significantly reduce the enhanced diffusion coefficients and actually simulate turbulence. This will require faster parallel computers and improved numerical methods and hopefully will happen within the next decade or two. In addition, sub-grid scale models need to be added to geodynamo models to better represent the heterogeneous anisotropic transport of heat, composition, momentum and possibly also magnetic field by the part of the turbulence spectrum that remains unresolved.

\section{References}

Busse, F.H., Homogeneous dynamos in planetary cores and in the laboratory, Ann. Rev. Fluid Mech. 32, 383-408, 2000.

Busse, F.H., Convective flows in rapidly rotating spheres and their dynamo action, Phys. Fluids 14, 1301-1314, 2002.

Christensen, U.R., J. Aubert, P. Cardin, E. Dormy, S. Gibbons, et al., A numerical dynamo benchmark, Phys. Earth Planet. Inter. 128 25-34, 2001.

Dormy, E., J.-P. Valet, and V. Courtillot, Numerical models of the geodynamo and observational constraints, Geochem. Geophys. Geosyst. 1, paper 2000GC000062, 2000.

Fearn, D.R., Hydromagnetic flow in planetary cores, Rep. Prog. Phys. 61, 175-235, 1998.

Gilman, P.A. and J. Miller, Dynamically consistent nonlinear dynamos driven by convection in a rotating spherical shell, Astrophys. J. Suppl. Ser. 46, 211-238, 1981.

Glatzmaier, G.A., Numerical simulations of stellar convective dynamos I. The model and the method, J. Comput. Phys. 55, 461-484, 1984. 
Glatzmaier, G.A., Geodynamo Simulations - How Realistic are They?, Ann. Rev. Earth Planet. Sci. 30, 237-257, 2002.

Glatzmaier, G.A., R.S. Coe, L. Hongre, and P.H. Roberts, The role of the Earth's mantle in controlling the frequency of geomagnetic reversals, Nature 401, 885-890, 1999.

Glatzmaier, G.A., and P.H. Roberts, A three-dimensional self-consistent computer simulation of a geomagnetic field reversal, Nature 377, 203-209, 1995.

Glatzmaier, G.A., and P.H. Roberts, Simulating the Geodynamo, Contemp. Phys., 38, 269-288, 1997.

Hollerbach, R., On the theory of the geodynamo, Phys. Earth Planet. Inter. 98, 163-185, 1996.

Jones, C.A., A. Longbottom, and R. Hollerbach, A self-consistent convection driven geodynamo model, using a mean field approximation, Phys. Earth Planet. Inter. 92, 119-141, 1995.

Kageyama, A., M. Ochi, T. Sato, Flip-flop transitions of the magnetic intensity and polarity reversals in the magnetohydrodynamic dynamo, Phys. Rev. Lett. 82, 5409-5412, 1999.

Kageyama, A., T. Sato, K. Watanabe, R. Horiuchi, T. Hayashi, et al., Computer simulation of a magnetohydrodynamic dynamo II, Phys. Plasmas 2, 1421-1431, 1995.

Kono, M. and P.H. Roberts, Recent geodynamo simulations and observations of the geomagnetic field, Rev. Geophys. 40, 4-1-53, 2002.

Kutzner, C. and U.R. Christensen, From stable dipolar towards reversing numerical dynamos, Phys. Earth Planet. Inter. 131, 29-45, 2002.

Roberts, P.H. and G.A. Glatzmaier, Geodynamo theory and simulations, Rev. Mod. Phys. 72, 1081-1123, 2000.

Sakuraba, A., and M. Kono, Effect of the inner core on the numerical solution of the magnetohydrodynamic dynamo, Phys. Earth Planet. Inter. 111, 105-121, 1999.

Sarson, G.R. and C.A. Jones, A convection driven dynamo reversal model, Phys. Earth Planet. Inter. 111, 3-20, 1999.

Zhang, K. and F.H. Busse, Finite amplitude convection and magnetic field generation in a rotating spherical shell, Geophys. Astrophys. Fluid Dyn. 4l, 33-53, 1988.

\section{Cross references}

Boussinesq and anelastic approximations

Core convection

Core dynamics: instabilities

Core-mantle boundary

Core-mantle boundary: implications for dynamics

Core-mantle coupling, thermal

Core-mantle coupling - electromagnetic

Core-mantle coupling - gravitational

Core-mantle coupling - topographic

Geodynamo

Geomagnetic dipole field

Inner core tangent cylinder 
Inner core, rotation

Magnetohydrodynamics

Reversals, theory

Seismic velocities of inner core

Solar dynamo

Spherical harmonics

Thermal wind

Turbulence in the Earth's core

Viscosity of the core

Westward drift 
Figure 1. A snapshot of the 3D magnetic field simulated with the Glatzmaier-Roberts geodynamo model and illustrated with a set of magnetic field lines. The axis of rotation is vertical and centered in the image. The field is complicated and intense inside the fluid core where it is generated by the flow; outside the core it is a smooth, dipole-dominated, potential field. [From Glatzmaier, 2002]

Figure 2. A sequence of snapshots of the longitudinally averaged magnetic field through the interior of the core and of the radial component of the field at the core-mantle boundary and at what would be the surface of the Earth, displayed at roughly 3000-year intervals spanning a dipole reversal from a geodynamo simulation. In the plots of the average field, the small circle represents the inner core boundary and the large circle is the core-mantle boundary. The poloidal field is shown as magnetic field lines on the left-hand sides of these plots (blue is clockwise and red is counter-clockwise). The toroidal field direction and intensity are represented as contours (not magnetic field lines) on the right-hand sides (red is eastward and blue is westward). Aitoff-Hammer projections of the entire coremantle boundary and surface are used to display the radial component of the field (with the two different surfaces displayed as the same size). Reds represent outward directed field and blues represent inward field; the surface field, which is typically an order of magnitude weaker, was multiplied by 10 to enhance the color contrast. [From Glatzmaier et al., 1999] 


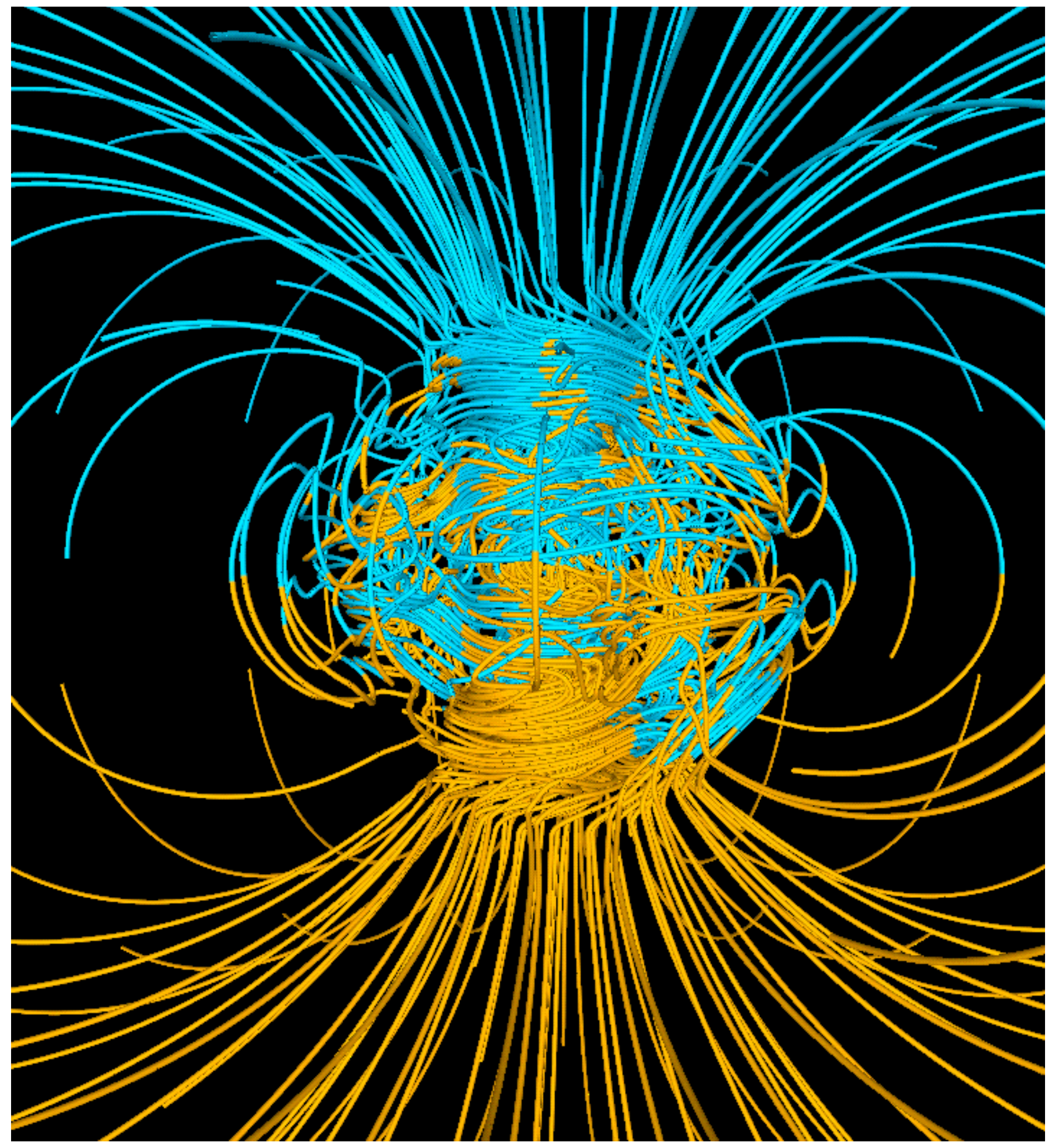




\section{Magnetic Field Reversal}

Four snapshots at 3000-year intervals

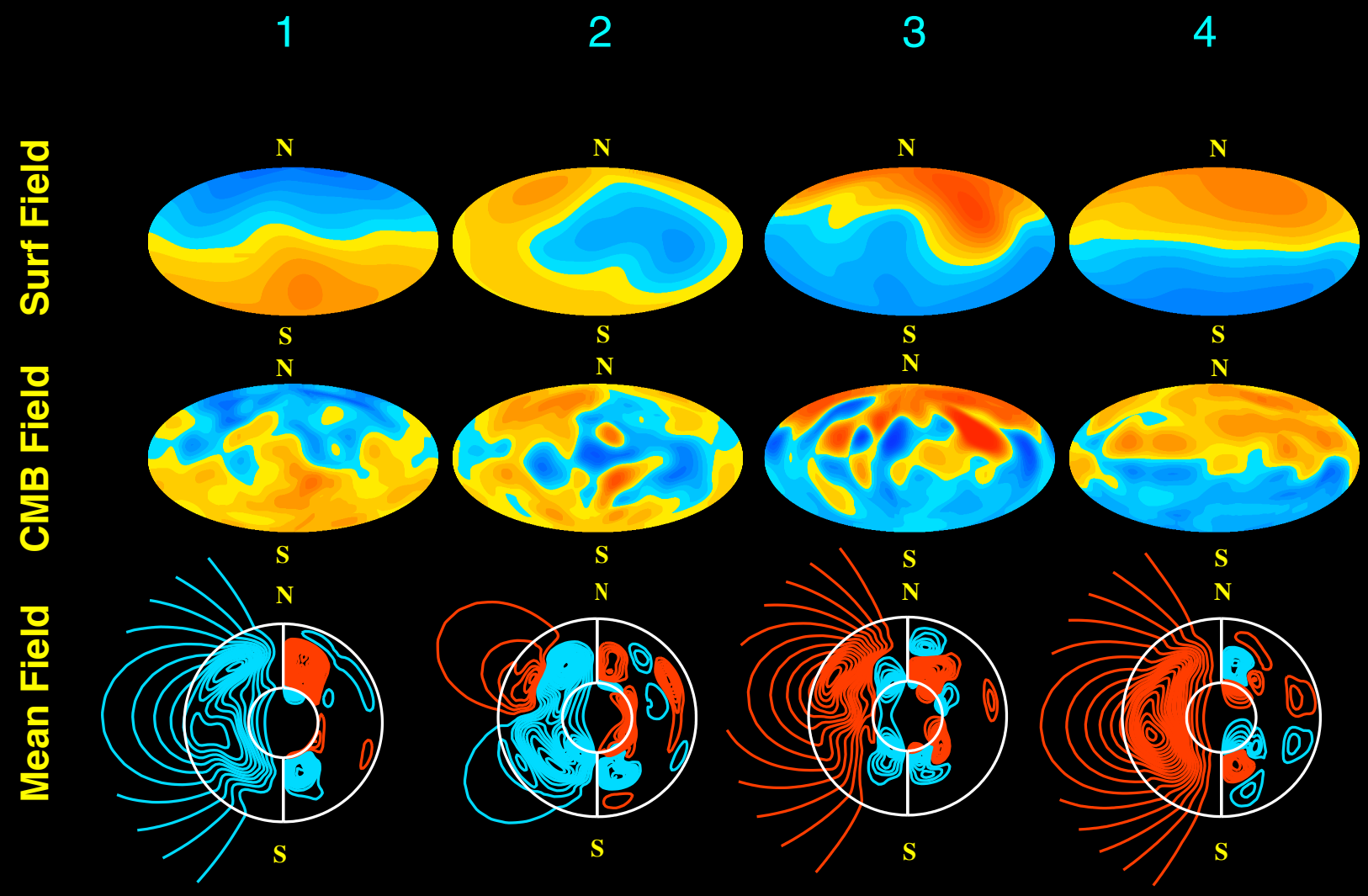

